\title{
Inteligencia Emocional y Hábitos de Estudio en Estudiantes de una Institución Publica
}

\author{
Emotional Intelligence and Study Habits in Students of a Public Institution
}

Inteligência emocional e hábitos de estudo em alunos de uma instituição pública

\section{ARTÍCULO ORIGINAL}

Yesica Elizabeth Garay Zorrilla

https://orcid.org/0000-0003-4614-0204

yescabu@hotmail.com

Investigadora Independiente
Leyla Paola Nuñez Guillen

https://orcid.org/0000-0002-4311-2518

lenunez@outlook.es

Investigadora Independiente

Recibido 28 de Setiembre 2021 | Arbitrado y aceptado 28 de Setiembre 2021 | Publicado en 01 Octubre 2021

\section{RESUMEN}

La investigación tuvo un enfoque cuantitativo, descriptiva de diseño no experimental de nivel correlacional, transaccional. Los instrumentos aplicados fueron el test conociendo mis emociones y el inventario de hábitos de estudio CASM, con validez de juicio de expertos y confiabilidad por alfa de Cronbach para inteligencia emocional 0,8,94 y para los hábitos de estudio ,829, la población fue de 75 estudiantes de $3^{\circ}$ de secundaria. Los resultados de $r$ Spearman obtuvo un $\mathrm{p}$-valor de 0,012 con lo que se afirma que la inteligencia emocional tiene una correlación significativa con los hábitos de estudio. El coeficiente de correlación de Spearman es $=, 290^{*}$ lo indica que la correlación es positiva bajo. lo que nos permite afirmar que mayor inteligencia emocional hay un mayor nivel de habito de estudio.

Palabras claves: Inteligencia Emocional, Hábitos de Estudio, Estudiantes, Técnicas, Adaptación.

\section{ABSTRACT}

The research had a quantitative, descriptive, non-experimental design of a correlational, transactional level. The instruments applied were the knowing my emotions test and the CASM study habits inventory, with validity of expert judgment and reliability by Cronbach's alpha for emotional intelligence $0.8,94$ and for study habits, 829, the population was than 753 rd year high school students. The results of $r$ Spearman obtained a p-value of 0.012 , which states that emotional intelligence has a significant correlation with study habits. Spearman's correlation coefficient is $=.290 *$ which indicates that the correlation is low positive. which allows us to affirm that greater emotional intelligence is a higher level of study habit.

Keywords: Emotional Intelligence, Study Habits, Students, Techniques, Adaptation.

\section{RESUMO}

A pesquisa teve um desenho quantitativo, descritivo e não experimental de nível correlacional e transacional. Os instrumentos aplicados foram o teste de conhecer minhas emoções e o inventário de hábitos de estudo CASM, com validade de julgamento de especialistas e confiabilidade pelo alfa de Cronbach para inteligência emocional $0,8,94$ e para hábitos de estudo, 829 , a população era superior a 75 alunos do $3^{\circ}$ ano do ensino médio. Os resultados de $r$ Spearman obtiveram um valor de $\mathrm{p}$ de 0,012 , o que afirma que a inteligência emocional tem uma correlação significativa com os hábitos de estudo. O coeficiente de correlação de Spearman é $=.290 *$ o que indica que a correlação é positiva baixa. o que nos permite afirmar que maior inteligência emocional é um nível superior de hábito de estudo.

Palavras-chave: Inteligência Emocional, Hábitos de Estudo, Alunos, Técnicas, Adaptação. 


\section{Introducción}

La inteligencia emocional está cada vez más presente en las aulas, que los estudiantes aprendan a identificar sus estados de ánimo, reconocer, gestionar y regular las emociones de ellos mismo y de los demás es lo que se busca en la actualidad. El desarrollo emocional de los estudiantes es un complemento indispensable para el desarrollo cognitivo y el buen desempeño académico, es así que las diferentes teorías psicológicas han formado distintos conceptos de la inteligencia emocional de origen biológico, neurológico, psicológico y cognitivo. Es así que tenemos a Gardner (1993) la inteligencia emocional puede formarse en el contexto cultural como un potencial biopsicologico para resolver problemas, para Salovey y Mayer (1997) lo presenta como la habilidad para emplear conocimiento de la vida y no solo táctico, así Goleman (1998) recoge estos aportes y plantea que la inteligencia emocional es la habilidad de la capacidad de ser inteligente utilizando nuestras emociones entender lo que sentimos y porque es importante como se sienten otros y usarlo en nuestra relación es totalmente necesaria para que funciones bien la vida en general y para la felicidad.

La adquisición de hábitos de estudios es importante porque ayuda a convertir estudiantes autónomos y responsables. Es importante que cuando los niños inician su formación escolar se le enseñe a tener esas costumbres de estudio y mientras más pequeños estén, mejor. Vicuña (1998) señala que los hábitos de estudio son un patrón conductual aprendido que suele presentarse mecánicamente y el individuo ya no tiene que decidir la forma de actuar ante la situación que se le presente. Es así que en esta investigación se busca conocer la relación existe entre la inteligencia emocional y hábitos de estudio en estudiantes.

\section{Metodología}

\section{Población}

75 estudiantes de $3^{\circ}$ de secundaria de la Instituciones Educativa publica "Cesar Vallejo".

\section{Instrumentos}

A. Inteligencia emocional: Conociendo mis emociones, se trata de un instrumento que evalúa diferentes áreas, está compuesta por 40 ítems en una escala tipo Likert de cinco puntos (Ruiz y Benítez, 2004). 
B. Hábitos de estudio: (CASM - 85), desarrollado por Vicuña Peri (1985) y con revisión en 1998, y en el 2005, que consta de 53 ítems distribuidos en 5 áreas: 1) ¿Cómo estudia Ud.?, constituido por 12 ítems; 2) ¿Cómo hace sus tareas?, constituido por 10 ítems. 3) ¿Cómo prepara sus exámenes?, constituido por 11 ítems. 4) ¿Cómo escucha las clases?, cuenta con 12 ítems. 5) ¿Qué acompaña sus momentos de estudio?, incluye 8 ítems.

\section{Procedimientos}

Para el tratamiento estadístico de datos seguimos el modelo que plantea por Hernández, et al. (2014) primero se seleccionó el software apropiado para el analizar los datos en este caso será el paquete estadístico para las ciencias sociales (SPSS 23) para la obtener los resultados se procedió de dos formas la estadística descriptiva donde se prende explorar y resumir los datos de la muestra a través de las siguientes herramientas: tablas de frecuencia, gráficos de barra y tablas cruzadas y la estadística inferencial donde se buscara generalizar los datos de la población a través de las siguientes herramientas: la estimación de parámetros con el estadístico Kolmogorov Smirnov para conocer si los datos son paramétricos o no paramétricos y según el resultado se escogerá se utilizara el estadístico R de Person si es que son paramétricos o el estadístico Rho de Spearman si es que no son paramétricos. Para conocer si existe alguna relación entre las variables inteligencia emocional y hábitos de estudio.

\section{RESULTADOS}

Tabla 1.

Correlación entre las variables inteligencia emocional y hábitos de estudio

Correlación

\begin{tabular}{|c|c|c|c|c|}
\hline & & & $\begin{array}{l}\text { Inteligencia } \\
\text { emocional }\end{array}$ & Hábitos de estudio \\
\hline \multirow{6}{*}{$\begin{array}{l}\text { Rho de } \\
\text { Spearman }\end{array}$} & \multirow{3}{*}{$\begin{array}{l}\text { Inteligencia } \\
\text { emocional }\end{array}$} & $\begin{array}{l}\text { Coeficiente de } \\
\text { correlación }\end{array}$ & 1,000 & ,290* \\
\hline & & Sig. (bilateral) &. &, 012 \\
\hline & & $\mathrm{N}$ & 75 & 75 \\
\hline & \multirow{3}{*}{$\begin{array}{l}\text { Hábitos de } \\
\text { estudio }\end{array}$} & $\begin{array}{l}\text { Coeficiente de } \\
\text { correlación }\end{array}$ & ,290* & 1,000 \\
\hline & & Sig. (bilateral) &, 012 & . \\
\hline & & $\mathrm{N}$ & 75 & 75 \\
\hline
\end{tabular}

*. La correlación es significativa en el nivel 0,05 (bilateral). 
Interpretación de la tabla 1: Con un p-valor de 0,012 la inteligencia emocional tiene una correlación significativa con los hábitos de estudio, pero la correlación es positiva bajo $=, 290^{*}$.

Tabla 2.

Correlación entre variable inteligencia emocional y la forma de estudio

Correlación

\begin{tabular}{|c|c|c|c|c|}
\hline & & & $\begin{array}{l}\text { Inteligencia } \\
\text { emocional }\end{array}$ & Forma de estudio \\
\hline \multirow{6}{*}{$\begin{array}{l}\text { Rho de } \\
\text { Spearman }\end{array}$} & \multirow{3}{*}{$\begin{array}{l}\text { Inteligencia } \\
\text { emocional }\end{array}$} & $\begin{array}{l}\text { Coeficiente de } \\
\text { correlación }\end{array}$ & 1,000 &,- 190 \\
\hline & & Sig. (bilateral) & . & ,103 \\
\hline & & $\mathrm{N}$ & 75 & 75 \\
\hline & \multirow{3}{*}{$\begin{array}{l}\text { Forma de } \\
\text { estudio }\end{array}$} & $\begin{array}{l}\text { Coeficiente de } \\
\text { correlación }\end{array}$ &,- 190 & 1,000 \\
\hline & & Sig. (bilateral) & 103 & . \\
\hline & & $\mathrm{N}$ & 75 & 75 \\
\hline
\end{tabular}

Interpretación de la tabla 2: Con un p-valor de 0,103 la inteligencia emocional no tiene una correlación significativa con la forma de estudio.

Tabla 3.

Correlación entre la variable inteligencia emocional y la resolución de tareas

Correlación

\begin{tabular}{|c|c|c|c|c|}
\hline & & & $\begin{array}{l}\text { Inteligencia } \\
\text { emocional }\end{array}$ & $\begin{array}{l}\text { Resolución de } \\
\text { tareas }\end{array}$ \\
\hline \multirow{6}{*}{$\begin{array}{l}\text { Rho de } \\
\text { Spearman }\end{array}$} & \multirow{3}{*}{$\begin{array}{l}\text { Inteligencia } \\
\text { emocional }\end{array}$} & $\begin{array}{l}\text { Coeficiente de } \\
\text { correlación }\end{array}$ & 1,000 &, $275^{*}$ \\
\hline & & Sig. (bilateral) & . &, 017 \\
\hline & & $\mathrm{N}$ & 75 & 75 \\
\hline & \multirow{3}{*}{$\begin{array}{l}\text { Resolución de } \\
\text { tareas }\end{array}$} & $\begin{array}{l}\text { Coeficiente de } \\
\text { correlación }\end{array}$ &, $275^{*}$ & 1,000 \\
\hline & & Sig. (bilateral) &, 017 & . \\
\hline & & $\mathrm{N}$ & 75 & 75 \\
\hline
\end{tabular}

*. La correlación es significativa en el nivel 0,05 (bilateral). 
Interpretación de la tabla 3: Con un p-valor de 0,017 la inteligencia emocional tiene una correlación significativa con la resolución de tareas, pero la correlación es positiva bajo $=, 275$.

\section{Tabla 4.}

Correlación entre la variable inteligencia emocional y la dimensión preparación para los exámenes

Correlación

\begin{tabular}{lllll}
\hline & & & $\begin{array}{l}\text { Inteligencia } \\
\text { emocional }\end{array}$ & $\begin{array}{l}\text { Preparación para los } \\
\text { exámenes }\end{array}$ \\
\hline & $\begin{array}{l}\text { Inteligencia } \\
\text { emocional }\end{array}$ & $\begin{array}{l}\text { Coeficiente de } \\
\text { correlación } \\
\text { Sig. (bilateral) }\end{array}$ & 1,000 &, $274^{*}$ \\
Rho de & & N & 75 &, 017 \\
Spearman & Preparación & Coeficiente de & 75 \\
& para los & correlación &, $274^{*}$ & 1,000 \\
& exámenes & Sig. (bilateral) &, 017 &. \\
& N & 75 & 75 \\
\hline
\end{tabular}

*. La correlación es significativa en el nivel 0,05 (bilateral).

Interpretación de la tabla 4: Con un p-valor de 0,017 la inteligencia emocional tiene una correlación significativa con la preparación para los exámenes, pero la correlación es positiva bajo $=, 274$.

\section{Tabla 5.}

Correlación entre la variable inteligencia emocional y la dimensión forma de escuchar

Correlación

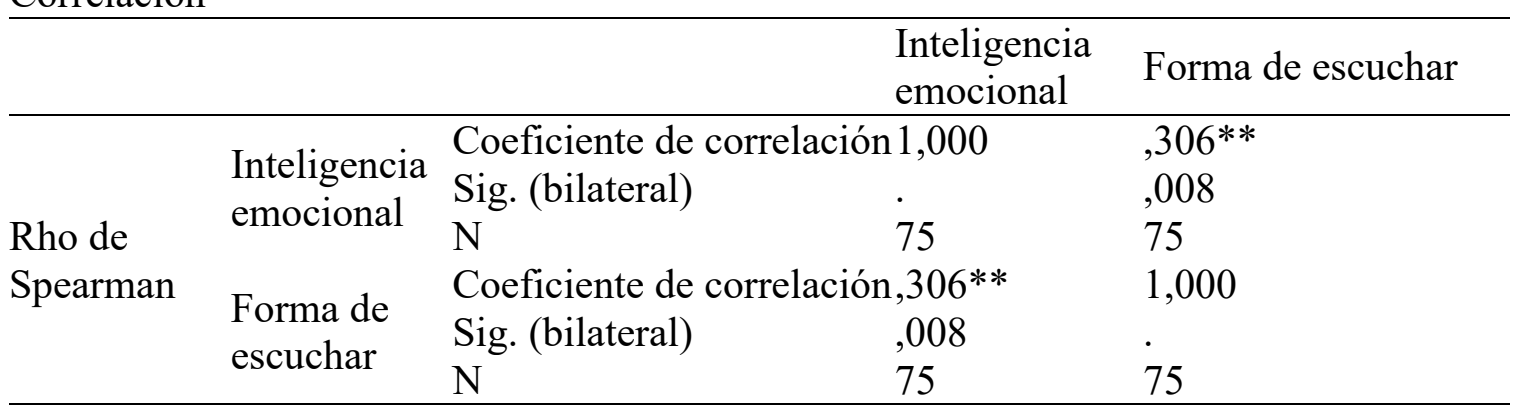

**. La correlación es significativa en el nivel 0,01 (bilateral). 
Interpretación de la tabla 5: Con un p-valor de 0,008 la inteligencia emocional tiene una correlación altamente significativa con la forma de escuchar, pero la correlación es positiva bajo $=, 306^{* *}$.

Tabla 6.

Correlación entre la variable inteligencia emocional y el acompañamiento al estudio

Correlación

\begin{tabular}{|c|c|c|c|c|}
\hline & & & $\begin{array}{l}\text { Inteligencia } \\
\text { emocional }\end{array}$ & $\begin{array}{l}\text { acompañamiento al } \\
\text { estudio }\end{array}$ \\
\hline \multirow{6}{*}{$\begin{array}{l}\text { Rho de } \\
\text { Spearman }\end{array}$} & \multirow{3}{*}{$\begin{array}{l}\text { Inteligencia } \\
\text { emocional }\end{array}$} & $\begin{array}{l}\text { Coeficiente de } \\
\text { correlación }\end{array}$ & 1,000 & ,112 \\
\hline & & Sig. (bilateral) & . & ,337 \\
\hline & & $\mathrm{N}$ & 75 & 75 \\
\hline & \multirow{3}{*}{$\begin{array}{l}\text { acompañamie } \\
\text { o al estudio }\end{array}$} & $\begin{array}{l}\text { Coeficiente de } \\
\text { t correlación }\end{array}$ & ,112 & 1,000 \\
\hline & & Sig. (bilateral) & ,337 & \\
\hline & & $\mathrm{N}$ & 75 & 75 \\
\hline
\end{tabular}

Interpretación de la tabla 6: Con un p-valor de 0,337 la inteligencia emocional no tiene una correlación significativa con el acompañamiento al estudio.

\section{Discusión}

La presente investigación tuvo como objetivo general establecer la relación que existe entre la inteligencia emocional y los hábitos de estudio en estudiantes de $3^{\circ}$ de secundaria de la Institución Educativa Publica Cesar Vallejo del distrito de San Juan de Lurigancho en el año académico 2018.

En ese sentido se encontró una baja relación significativa entre la inteligencia emocional y los hábitos de estudio en los estudiantes de $3^{\circ}$ de secundaria al haberse hallado una correlación de ,290*; nos permite afirmar que mayor inteligencia emocional hay un mayor nivel de habito de estudio. Similar resultado encontró Sarabia (2012), en la investigación hábitos de estudio y rendimiento académico en los estudiantes de cuarto a séptimo de básica de la escuela, donde concluye que existe una relación significativa entre dichas variables los estudiantes que sacaron buen puntaje en el rendimiento académico expresaron excelentes hábitos de estudio. Así también Sánchez (2015), en su 
investigación halló relación significativa y positiva entre los niveles de hábitos de estudio y los niveles del rendimiento académico en estudiantes del tercer año de secundaria de una Institución Educativa, el contraste de los antecedentes se debe quizás a la similitud de la población y al marco teórico de la variable inteligencia emocional, según la teoría de Salovey y Mayer (1990) la inteligencia emocional es un conjunto de procesos mentales como la evaluación, expresión, regulación y la utilización de la emoción que formando hábitos saludables en las personas esto estaría corroborado por esta y otras investigaciones.

A nivel específico, el objetivo 1 fue determinar la relación que existe entre la inteligencia emocional y la forma de estudio en estudiantes de $3^{\circ}$ de secundaria de la Institución Educativa Publica Cesar Vallejo. Al contrastar la hipótesis se obtuvo un coeficiente de correlación de 0,103 y una significancia de -,190 lo que indica que no existe relación entre la inteligencia emocional y la forma de estudio en estudiantes.

De la misma manera en el objetivo específico 2 fue determinar la relación que existe entre la inteligencia emocional y la resolución de tareas en estudiantes de $3^{\circ}$ de secundaria de la Institución Educativa Publica Cesar Vallejo. Al contrastar la hipótesis se obtuvo un coeficiente de correlación de $=0,017$ y una significancia de $=, 275$ lo que indica que no existe relación entre la inteligencia emocional y la resolución de tareas en los estudiantes. También el en objetivo específico 3 fue determinar la relación que existe entre la inteligencia emocional y la preparación para los exámenes en estudiantes de $3^{\circ}$ de secundaria de la Institución Educativa Publica Cesar Vallejo. Al contrastar la hipótesis se obtuvo un coeficiente de correlación de $=0,017$ y una significancia de $\mathrm{p}$-valor $=, 274$ que indica que no existe relación entre la inteligencia emocional y la preparación para los exámenes en los estudiantes. Y por último el nivel específico, el objetivo 4 fue determinar la relación que existe entre la inteligencia emocional y la forma de escuchar en estudiantes de $3^{\circ}$ de secundaria de la Institución Educativa Publica Cesar Vallejo. Al contrastar la hipótesis se obtuvo un coeficiente de correlación de 0,008 y una significancia de ,306** que indica que no existe relación en la inteligencia emocional y el acompañamiento al estudio.

Los cuatro objetivos dieron similares resultados que no existen relación significativa entre la inteligencia emocional y la forma de estudiar, la resolución de tareas, la preparación para los exámenes y la forma de escuchar. Similares resultados fueron 
encontrados por Cassinda, et al. (2016), en su investigación indicó que la inteligencia emocional: Su relación con el rendimiento académico en preadolescentes de la Escuela, donde concluye que no existe relación entre una variable y dimensión forma de estudio. Por otro lado, según la teoría de Vicuña (1998) sobre hábitos de estudio menciona que la forma de estudiar es una técnica que realiza el estudiante para comprender los puntos más importantes de una actividad académica, la resolución de tareas es la conducta que realiza el estudiante frente al desarrollo del trabajo académico, la preparación para los exámenes es la acción que el estudiante realiza antes de dar un examen y la forma de escuchar la clase es como el estudiante compre la clase, todas estas dimensiones no necesariamente se dará por la inteligencia emocional que posee los estudiantes, sino talvez por el estilo de aprendizaje que ha desarrollado o por el tipo de personalidad que tiene el estudiante, la investigación de Parodi, et al. (2017) indicó que la inteligencia emocional y personalidad de estudiantes de educación secundaria confirmaría de cierta forma este resultado donde manifiesta que la inteligencia emocional varían según los rasgos de personalidad.

Por el lado de la inteligencia emocional Goleman (1995) mencionó dos principales modelos y de ellos se derivan docenas de variaciones, entre estas podemos encontrar el modelo de rasgo, el cual se basa en los patrones de conducta y el modelo de procesamiento de la información, el cual habla directamente de la relación entre la inteligencia emocional y la cognición, entonces no dice que las emociones y la cognición pueden estar separados, es así que las acciones y conductas de los estudiantes como la forma de estudiar, resolución de tareas, la preparación para los exámenes y la forma de escuchar la clase va depender del tipo de estilo de aprendizaje más que la inteligencia emocional que pueda tener el estudiante es por ello que no se encuentra relación entre los objetivos.

Y por último a nivel específico, el objetivo 5 fue determinar la relación que existe entre la inteligencia emocional y el acompañamiento al estudio en estudiantes de $3^{\circ}$ de secundaria de la Institución Educativa Publica Cesar Vallejo. Al contrastar la hipótesis se obtuvo un coeficiente de correlación de $=0,337$ y una significancia de $=, 112$ que indica que existe baja relación entre la inteligencia emocional y el acompañamiento al estudio en los estudiantes. De la misma manera Wilson (2018) en la investigación hábitos de estudio y rendimiento académico en estudiante de secundaria los resultados dieron que existe una baja relación entre los hábitos de estudio y el rendimiento académico. También Lopez y Jimenez (2015) en la investigación hábitos de estudio y rendimiento académico 
en estudiante de secundaria los resultados dieron que existe una relación entre los hábitos de estudio y el rendimiento académico. En estudiante de quinto de secundaria de una Institución Educativa Publica, la similitud de resultados de estas investigaciones se deba quizá por la población por los instrumentos de evaluación utilizados, en la teoría tenemos a Thorndike (1920) indicó la inteligencia emocional se basa en la actitud con que cada persona enfrenta su entorno, y por lado de los hábitos de estudio Vicuña (1998) indicó que es la acción que el estudiante realiza durante el estudio. Los antecedentes de investigación y las teorías confirmarían el resultado encontrados por esta investigación.

\section{Conclusiones}

1. Existe relación entre la inteligencia emocional y los hábitos de estudio en estudiantes de $3^{\circ}$ de secundaria de la Institución Educativa Publica Cesar Vallejo del distrito de San Juan de Lurigancho en el año académico 2018

2. No existe relación significativa entre la inteligencia emocional y la forma de estudio en estudiantes de $3^{\circ}$ de secundaria de la Institución Educativa Publica Cesar Vallejo del distrito de San Juan de Lurigancho en el año académico 2018

3. Existe relación significativa entre la inteligencia emocional y la resolución de tareas en estudiantes de $3^{\circ}$ de secundaria de la Institución Educativa Publica Cesar Vallejo del distrito de San Juan de Lurigancho en el año académico 2018

4. Existe relación significativa entre la inteligencia emocional y la preparación para los exámenes en estudiantes de $3^{\circ}$ de secundaria de la Institución Educativa Publica Cesar Vallejo del distrito de San Juan de Lurigancho en el año académico 2018

5. Existe relación significativa entre la inteligencia emocional y la forma de escuchar la clase en estudiantes de $3^{\circ}$ de secundaria de la Institución Educativa Publica Cesar Vallejo del distrito de San Juan de Lurigancho en el año académico 2018

6. No existe relación significativa entre la inteligencia emocional y el acompañamiento al estudio en estudiantes de $3^{\circ}$ de secundaria de la Institución Educativa Publica Cesar Vallejo del distrito de San Juan de Lurigancho en el año académico 2018 


\section{Referencias}

Cassinda, M., Chingombe, A., Angulo, L., y Guerra, V. (2016). “Inteligencia emocional: Su relación con el rendimiento académico en preadolescentes de la Escuela 4 de abril, de Io ciclo, Angola”. (tesis de maestría). Recuperado de: https://revistas.ucr.ac.cr/index.php/educacion/article/view/22713.

Gardner, H. (1993). Multiple intelligences: The theory in practice. New York: Basic Books, 1993, 320 p. ISBN: 978-0465047680.

Goleman, D. (1995). Emotional Intelligence. New York: Bantam.

Goleman, D. (1998). Trabajando con inteligencia emocional. New York: Bantam Books, ISBN: 978-0553378580.

Lopez, S. y Jiménez, G. (2015). “Hábitos de estudio y rendimiento académico de las estudiantes de quinto grado de secundaria de una Institución Educativa Pública de Chiclayo". (tesis de pregrado). Recuperado de: https://docplayer.es/85235009Carrera-profesional-de-psicologia.html.

Parodi, A., Belmonte, V., Ferrándiz, C., y Ruiz, M. (2017). "La relación entre la inteligencia emocional y la personalidad en estudiantes de educación secundaria”. (Tesis de maestría). Recuperado de: http://www.infad.eu/RevistaINFAD /OJS/index.php/IJODAEP/article/view/926/805.

Ruiz y Benítez (2004). Test Conociendo mis Emociones. Perú: Departamento Psicopedagógico.

Salovey, P. y Mayer, J. D. (1997). Desarrollo educativo e inteligencia emocional. New York: Basic Books.

Salovey, P., Mayer, J. y Caruso, D. (2002). "The positive psychology of emotional intelligence”. The handbook of positive psychology. New York: Oxford University Press.

San Martín, R. (2009). Los malos hábitos a la hora de estudiar. Diario La Nación, edición electrónica. Recuperado de: http://www.lanacion.com.ar/615807-los-maloshabitos-ha-la-hora-de-estudiar. 
Sánchez, S. (2015). "Relación entre hábitos de estudios y rendimiento académico en estudiantes del tercer año de secundaria de una Institución Educativa del distrito de San Martin De Porres, provincia de Lima, año 2015”. (tesis de maestría). Recuperado de: http://repositorio.uap.edu.pe/handle/uap/4754?mode=full.

Thorndike, E. (1920). Inteligencia y sus usos. Recuperado de: file://C:/Users/ USISE\%7D /Downloads/38963-Texto\%20del\%20art\%C3\%ADculo-47025-1-1020120410.pdf.

Trujillo, M. y Rivas, A. (2005) Orígenes, evolución y modelos de inteligencia emocional. Innovar, Revista de Ciencias Administrativas y Sociales.

Vicuña, L. (1998). Inventario de hábitos de estudio. Lima: centro de desarrollo e investigación psicología.

Vicuña, L. (2005). Inventario de hábitos de estudio. Lima: centro de desarrollo e investigación psicología.

Wilson, S. (2018). "Los Hábitos de Estudio y el Rendimiento Académico en los estudiantes de quinto, sexto y séptimo de educación general básica en la Unidad Educativa “Avelina Lasso de Plaza”, periodo 2016-2017” (tesis de pregrado). Recuperado de: http://www.dspace.uce.edu.ec/bitstream/25000/14163/1/T-UCE0010-PEO039-2018.pdf. 